\title{
Research on Deployment Height of Sensing Reconnaissance Submunition
}

\author{
Li Jun ${ }^{\mathrm{a}}$, Li Yun ${ }^{\mathrm{b}}$, Wang $\mathrm{Qi}^{\mathrm{c}}$, Zou Jun ${ }^{\mathrm{d}}$ \\ New Star Research Institute of Applied Technology, Hefei, 230031, China \\ aemail: ljazq1314@163.com, bemail:Iyhfpy@163.com \\ cemail:1715026200@qq.com, demail:2249861737@qq.com
}

Keywords: sensing reconnaissance submunition; deployment height; MATLAB/Simulink

\begin{abstract}
The Parachute deployment height of sensing reconnaissance submunition has great effect on its landing state and precision; to determine deployment height of sensing reconnaissance submunition, using MATLAB/Simulink simulation platform, the motion of Parachute-Submunition System was simulated by numerical method, the results are in good agreement with the experimental data. And considering the wind, charge number and firing angel etc, the height of the reconnaissance submunition was simulated, the result indicate: the deployment height of $1 \#$ charge should be in $175 \sim 250 \mathrm{~m}$, the deployment height of $6 \#$ charge should be in $150 \sim 225 \mathrm{~m}$. The method have some certain guiding value for deciding the deployment height of other Parachute landing submunition.
\end{abstract}

\section{Introduction}

Sensing reconnaissance submunition is a new kind of munition which is based on shell as a launch vehicle, the submunition is delivery to the designated area through cluster, to complete a few special reconnaissance mission. In order to avoid the damage of precision sensor equipment to work properly, it requires submunition landing speed not too high, and falling angel also has requirements. If the deployment height is a known scope, submunition landing flight reaches stable state. If deployment height is too high, level speed is larger, the displacement range is larger, and the submunition falling time is longer, in the process of whereabouts are greatly influenced by the meteorological conditions, where it is difficult to control. If the deployment height is too low, although the submunition flight time and flight distance is reduced, and the submunition is less influenced by conditions, the speed is faster after submunition thrown from the dispenser cabin, ballistic parameters change drastically, the submunition has not yet reached a stable state on landing, thus it affects the normal work of the submunition. Therefore, it is very important to the choice of the right deployment height for the stability of the submunition and accurate control of placement.

\section{Built of Ballistic Model of Falling Ammunition}

In the process of dispensing, submunition flights free to the target area with shell, then the bottom of the shell opens at a certain height, parachute is threw from the bottom of shell, submunition is dispensed from the shell and rapid deceleration under the action of parachute, and submunition which is hanged under the parachute lands slowly in the reconnaissance area. Therefore, the trajectory is a complex composite ballistic.

Due to parachute-opening process is complicated, time is very short, and when the submunition state changes very little, so scatter and deploying process aren't considered. Therefore, submunition and parachute can be considered as Parachute-Submunition System. The movement process of the submunition is basically the same in the falling stage, which undergo deceleration stage and steady falling stage. Suppose not consider that the parachute is breathing and breathable, the axis of parachute and submunition always coincide in the process of inflation, and does not consider the elastic of the rope.

2.1 Deceleration Stage 
Since the resistance of parachute is very big after opening, so there is relative motion between the parachute and reconnaissance submunitions. If the parachute and reconnaissance submunition are considered as a whole, there will be large error in ballistic model. Therefore, it is necessary to select the parachute and reconnaissance submunition as the research objects ${ }^{[1]}$.

On the basis of the momentum theorem $m_{d} \frac{\mathrm{d} \vec{v}}{\mathrm{~d} t}=\sum \vec{F}_{i}$, the center of mass motion differential equations of submunition are built:

$$
\left\{\begin{array}{l}
\frac{d x}{d t}=v \cos \psi \cos \theta \\
\frac{d y}{d t}=-v \cos \theta \sin \psi \\
\frac{d z}{d t}=v \sin \theta \\
\frac{d v}{d t}=g \cos \psi \sin \theta-\frac{R_{d}+N}{m_{d}} \\
\frac{d \theta}{d t}=\frac{g \cos \theta}{v \cos \psi} \\
\frac{d \psi}{d t}=-\frac{g \sin \psi \sin \theta}{v}
\end{array}\right.
$$

$v$ is speed of fall, $\theta$ is trajectory inclination angle, $\psi$ is trajectory declination angle, $R_{d}$ is aerodynamic resistance of submunition, $N$ is the pulling force of the parachute to submunition, $m_{d}$ is the quality of submunition.

The equation of parachute is built:

$\frac{d}{d t}\left[\left(m_{p}+m_{f}\right) v\right]=F_{k}-R_{p}+m_{p} g \cos \psi \sin \theta$

$m_{p}$ is the quality of parachute, $R_{p}$ is the aerodynamic resistance of parachute, $F_{k}$ is opening loading, $m_{f}$ is added mass.

Base on the document【2】, the change rule of air resistance coefficient of parachute in the inflation process is:

$$
\begin{aligned}
& C_{A}=\left\{\begin{array}{cl}
K s \quad, \quad & s_{0} \leq s \leq s_{1} \\
C_{A_{1}}+\beta\left(s-s_{1}\right)^{4}, & s_{1} \leq s \leq s_{m}
\end{array}\right. \\
& C_{A_{1}}=0.04 C_{A_{f}}, s_{1}=\lambda D_{p}, K=C_{A_{1}} / s_{1}, \\
& \beta=\left(C_{A f}-C_{A_{1}}\right) /\left(s_{m}-s_{1}\right)^{4}, \lambda=1.74, \alpha \in[6,7] \\
& m_{f}=K_{f} \rho C_{A}^{3 / 2}, C_{A} \text { is the transient resistance coefficient of parachute system, } F_{k} \text { is opening }
\end{aligned}
$$
shock. Other parameter is referenced at the document 【2】.

Uniting equation (1), (2), and (3), the movement equations of Parachute-Submunition System are: 


$$
\left\{\begin{array}{l}
\frac{d x}{d t}=v \cos \psi \cos \theta \\
\frac{d y}{d t}=v \sin \theta-v \cos \theta \sin \psi \\
\frac{d z}{d t}=v \sin \theta \\
\frac{d v}{d t}=g \cos \psi \sin \theta-\frac{R_{D}+N}{m_{D}} \\
\frac{d \theta}{d t}=\frac{g \cos \theta}{v \cos \psi} \\
\frac{d \psi}{d t}=-\frac{g \sin \psi \sin \theta}{v} \\
F_{k}=R_{p}+v \frac{d m_{f}}{d t}+\left(m_{p s}+m_{f}\right) \frac{d v}{d t}-m_{p s} g \cos \psi \sin \theta \\
m_{f}=K_{f} \rho C_{A}^{3 / 2} \\
\frac{d m_{f}}{d t}=\frac{3}{2} K_{f} \rho C_{A}^{1 / 2} \frac{d C_{A}}{d s} \quad, \quad s_{0} \leq s \leq s_{1} \\
C_{A}=\left\{\begin{array}{cl}
K s & \quad s_{0} \leq s \leq s_{1} \\
C_{A_{1}}+\beta\left(s-s_{1}\right)^{4} & , \quad s_{1} \leq s \leq s_{m}
\end{array}\right. \\
\frac{d C_{A}}{d t}=\left\{\begin{array}{cc}
K & s_{1} \leq s \leq s_{m} \\
4 \beta\left(s-s_{1}\right)^{3}
\end{array}\right.
\end{array}\right.
$$

The projection area of the parachute is the largest at the end of the parachute inflation.

2.2 Steady Falling Stage

Parachute-Submunition System continues to descend as a whole under the action of aerodynamic drag when parachute is inflatable full, until the system reaches balance, then the descent velocity basically keeps constant.

The movement equations of Parachute-Submunition System are:

$$
\left\{\begin{array}{l}
\frac{d v_{x}}{d t}=-\frac{1}{2\left(m_{p}+m_{d}\right)} \rho v\left(S_{d} C_{d 2}+S_{p} C_{p 2}\right)\left(v_{x}-w_{x}\right) \\
\frac{d v_{x}}{d t}=-\frac{1}{2\left(m_{p}+m_{d}\right)} \rho v\left(S_{d} C_{d 2}+S_{p} C_{p 2}\right) v_{y}-g \\
\frac{d v_{x}}{d t}=-\frac{1}{2\left(m_{p}+m_{d}\right)} \rho v\left(S_{d} C_{d 2}+S_{p} C_{p 2}\right)\left(v_{z}-w_{z}\right) \\
\frac{d x}{d t}=v_{x}, \frac{d y}{d t}=v_{y}, \frac{d z}{d t}=v_{z}
\end{array}\right.
$$

$S_{d}$ is effective area of submunition, $C_{d 2}$ is the resistance coefficient of submunition, $S_{p}$ is effective area of parachute, $C_{p 2}$ is the resistance coefficient of parachute, and other parameters are the same as above.

\section{Simulating Calculation}

\subsection{The Establishment to Simulation Model}

The simulation tool MATLAB/Simulink is an interactive dynamic system modeling, simulation and analysis of integrated development software, with a powerful numerical calculation capacity, the users can also customize the function. It provides a friendly graphical user interface, get rid of the tedious code programming. Therefore, the article selects MATLAB/Simulink simulation tool to simulate landing process.

In order to describe the parachute process, using ISA Atmosphere Model in Aerospace Blockest which provides descriptions of the atmospheric environment, and Math Operation which reflect the relationship between the variables, and other modules in Simulink module library, to set up the parachute model. 
As the landing process of submunition includes two stages, the system simulation is divided into two parts according to time. The results of the former part is considered as the starting conditions of latter part. Firstly, the Parachute-Submunition System simulation model in the deceleration stage is set up, as shown in figuer1. Secondly, considering the Parachute-Submunition System as a whole, and three degrees of freedom simulation in steady falling stage is presented, as shown in figure 2.

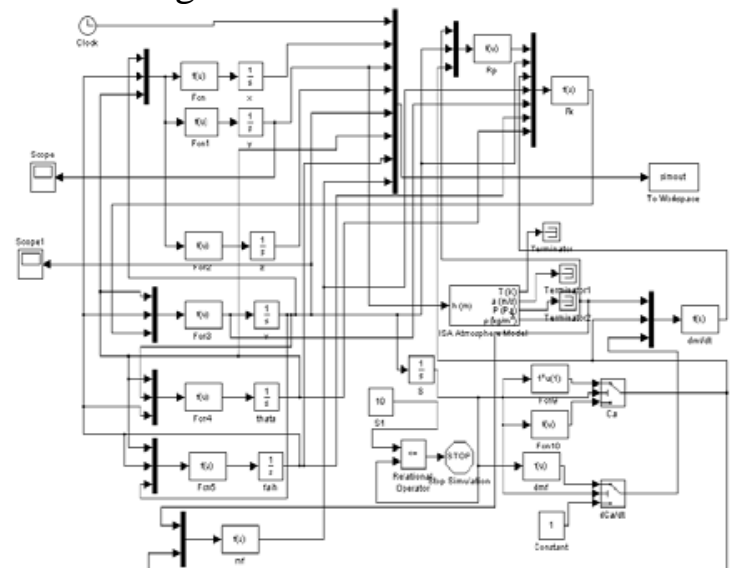

Fig.1 Simulation chart in deceleration stage

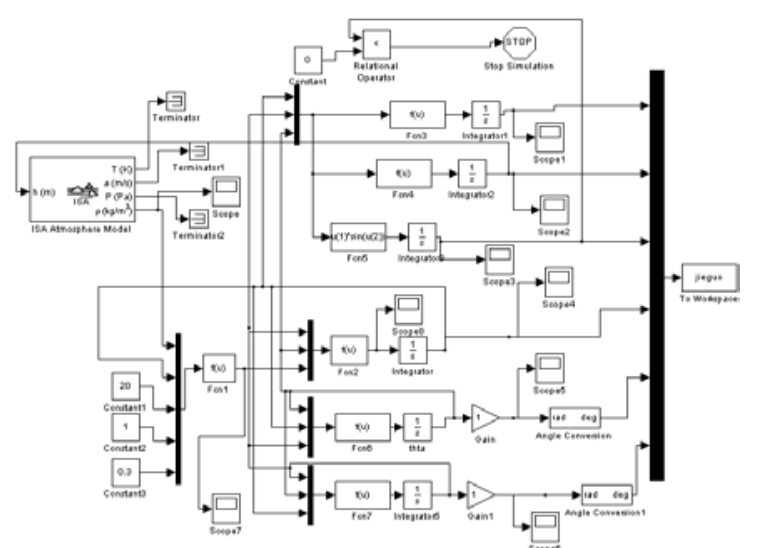

Fig.2 Simulation chart in steady falling stage

\subsection{Simulation Result}

With a certain type sensor submunition as an example, the simulation calculation is studied using the simulation process as above, and with the same initial conditions the results are compared of a live firing test results in the northeast, the results are shown in table1.

Tab.1 Corresponding Submunition Data in Different Scatter Height

\begin{tabular}{|c|c|c|c|c|c|c|}
\hline \multirow{2}{*}{$\begin{array}{c}\text { Scatter } \\
\text { Height } \\
(\mathrm{m})\end{array}$} & \multicolumn{2}{|c|}{$\begin{array}{c}\text { Hang Time of Submunition } \\
(\mathrm{s})\end{array}$} & $\begin{array}{c}\text { The Landing Speed of } \\
\text { Submunition } \\
(\mathrm{m} / \mathrm{s})\end{array}$ & \multicolumn{2}{c|}{$\begin{array}{c}\text { The Flying Distance of } \\
\text { Submunition } \\
(\mathrm{m})\end{array}$} \\
\cline { 2 - 7 } & $\begin{array}{c}\text { experimental } \\
\text { value }\end{array}$ & $\begin{array}{c}\text { calculated } \\
\text { value }\end{array}$ & $\begin{array}{c}\text { experimental } \\
\text { value }\end{array}$ & $\begin{array}{c}\text { calculated } \\
\text { value }\end{array}$ & $\begin{array}{c}\text { experimental } \\
\text { value }\end{array}$ & $\begin{array}{c}\text { calculated } \\
\text { value }\end{array}$ \\
\hline 165 & 17.1 & 16.3 & 11.0 & 11.4 & 128 & 133 \\
\hline 206 & 20.1 & 19.1 & 11.7 & 11.2 & 197 & 189 \\
\hline 225 & 21.3 & 20.6 & 11.4 & 10.7 & 246 & 241 \\
\hline
\end{tabular}

From table 1, we can see that the error of calculated value and experimental value is very little, so the simulation is feasible, the ballistic model of Parachute-Submunition System is effective.

\section{The Deployment Height}

According to the established simulation model, the distance and angle error which are caused by the vertical wind and horizontal wind of 5m/s using the charge of NO.1 and NO.6 are analyzed, as shown in table2. The landing speed and ballistic inclined angle has been simulated when the deployment height is 75 meters to 300 meters, as shown in figure 4 and figure 5 . In order to achieve the submunition to work normally and precision scatter, landing speed is less than $15 \mathrm{~m} / \mathrm{s}$, ballistic inclined angle is greater than $80^{\circ}$, and the deviation caused by wind is limited within the range of 80 meters. Based on the results in table 2 and figure 3, figure 4, we can see that the deployment height should be in the range of 175 250 meters using charge of NO.1, and the deployment height should be in the range of $150 \sim 225$ meters using charge of NO.6.

Table 2 The Distance and Angle in Different Height

\begin{tabular}{|c|c|c|c|c|}
\hline \multirow{2}{*}{ Charge Number } & \multicolumn{2}{|c|}{$1 \#$} & \multicolumn{2}{|c|}{$6 \#$} \\
\cline { 2 - 5 } & $\begin{array}{c}\text { Distance } \\
(\mathrm{m})\end{array}$ & $\begin{array}{c}\text { Angle } \\
\left({ }^{\circ}\right)\end{array}$ & $\begin{array}{c}\text { Distance } \\
(\mathrm{m})\end{array}$ & $\begin{array}{c}\text { Angle } \\
\left({ }^{\circ}\right)\end{array}$ \\
\hline
\end{tabular}




\begin{tabular}{|c|c|c|c|c|}
\hline 75 & 8.7 & 9.2 & 10.6 & 11.7 \\
\hline 100 & 17.4 & 18.5 & 20.6 & 21.2 \\
\hline 125 & 21.1 & 26.7 & 29.5 & 31.6 \\
\hline 150 & 27.8 & 36.3 & 41.3 & 41.2 \\
\hline 175 & 36.3 & 45.0 & 53.3 & 55.7 \\
\hline 200 & 42.7 & 53.8 & 66.5 & 70.2 \\
\hline 225 & 64.2 & 66.2 & 71.1 & 79.4 \\
\hline 250 & 67.1 & 70.2 & 76.4 & 87.6 \\
\hline 275 & 74.9 & 81.1 & 80.9 & 87.8 \\
\hline 300 & 84.3 & 87.4 & 93.8 & 95.1 \\
\hline
\end{tabular}

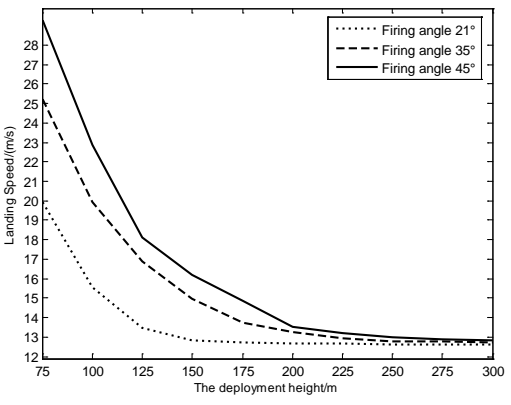

NO.1

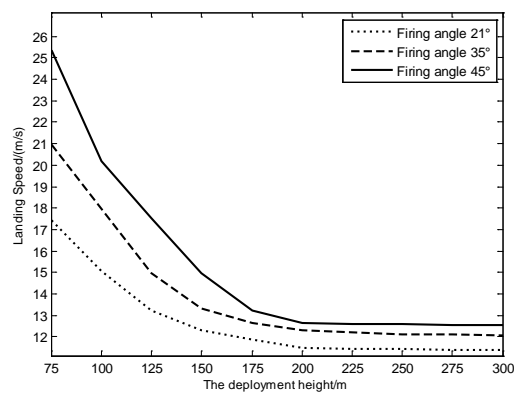

NO.6

Fig.3 The Relationship of Landing Speed and Deployment Height

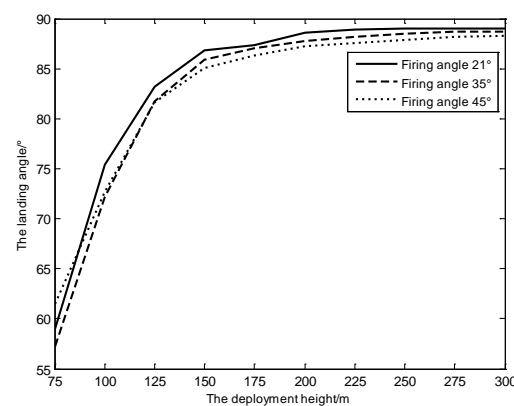

NO.1

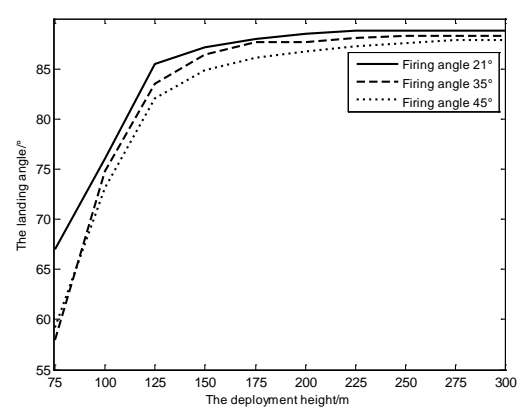

NO.6

Fig.4 The Relationship of Landing Inclination Angle and Deployment Height

\section{Conclusion}

In this paper, based on the ballistic model of Parachute-Submunition System, using MATLAB/Simulink simulation platform, and considering the vertical wind, horizontal wind, charge number and firing angel etc, the motion of Parachute-Submunition System is simulated. The calculation results shown as follows:

(1)In this paper, the model can accurately response sensing reconnaissance submunition flighting in falling stage, the results are in good agreement with the experimental data.

(2)In practical shooting, the select of deployment height should comprehensively consider the effect of various factors. On the premise of guarantee the submunition was basically stable in falling process, as far as possible selecting lower deployment height, to reduce the hang time of the mission equipment, avoid too much influence by external environment, so as to achieve the goal of improving the accuracy of point of fall.

(3)In this paper, the research methods on the analysis of the selecting of deployment height have a guiding significance for other falling submunition. 


\section{References}

[1] Yang Qiren. Shrapnel Fight Dynamics [M].Beijing: National Defence of Industry Press, 1999.82-140

[2] Li Dayao. Using Rigid Body-particle Trajectory Model Method to Calculate Projectileparachute System Basic Equations [J].Spacecraft Recovery \& Remote Sensing,1995,16(3):4-16

[3] Liu Yuwen, Ma Jianhua. The Ballistic Model Establishment And Simulation Of Sensor

Scouting Shell[J]. Xi'an: Journal Of Projectiles Rockets Missiles And Guidance, 2009, 29(5):177-178

[4] Xue Dingyu. MATLAB/Simulink System Simulation and Application[M]. Beijing: Tsinghua University Press, 2007

[5] Guo Rui etc. Dynamics Model for Terminal-Sensitive Submunition in Deceleration Stage [J].Journal of Projectiles, Rockets, Missiles and Guidance,2005(4):186-189 